This article is published with open access at Springerlink.com

\title{
On functions that are approximate fixed points almost everywhere and Ulam's type stability
}

\author{
Anna Bahyrycz, Janusz Brzdęk, Eliza Jabłońska and \\ Jolanta Olko
}

\begin{abstract}
In this paper, we investigate functions that are approximate fixed points of some (possibly nonlinear) operators almost everywhere, with respect to some ideals of sets. We prove that (under suitable assumptions) there exist fixed points of the operators that are "near" those functions. The results are applied to obtain some general stability results of Ulam's type almost everywhere; in particular, for the polynomial functional equation.
\end{abstract}

Mathematics Subject Classification. Primary 39B82; Secondary $47 \mathrm{H} 10$.

Keywords. Ulam's type stability, fixed point almost everywhere, approximate fixed point, nonlinear operator.

\section{Introduction}

This paper has been motivated mainly by the fixed point results obtained in $[5,6,10]$ (we recall them in what follows, in a bit generalized forms) and their applications in Ulam's type stability. Let us remind that the interest in investigation of that type of stability has been stimulated by a problem of S. M. Ulam (cf. $[14,20]$ ) and several papers that appeared afterwards (for more details and the references concerning that subject, we refer the reader to $[1,3,4,11,12,15,16,17])$.

There arises a natural question whether some outcomes, similar to those in $[5,6,10]$, can be obtained if some suitable conditions are assumed to hold only almost everywhere, with respect to some ideals of sets.

Denote by $\mathbb{N}, \mathbb{N}_{0}$ and $\mathbb{R}_{+}$the sets of positive integers, nonnegative integers and nonnegative real numbers, respectively. Let $X$ be a nonempty set. A family $\mathcal{I} \subset 2^{X}$ is a $\sigma$-ideal in $X$ if it contains the empty set, subsets and countable unions of its elements. We say that a property $p(x)$ holds $\mathcal{I}$-almost everywhere in $X$ (I $\mathcal{I}$-(a.e.) for abbreviation) if there exists a set $A \in \mathcal{I}$ such 
that $p(x)$ is valid for all $x \in X \backslash A$. We say that $g: X \rightarrow Y$ is an $\mathcal{I}$-unique function satisfying some conditions if $\widetilde{g}(x)=g(x) \mathcal{I}$-(a.e.) for each $\widetilde{g}: X \rightarrow Y$ satisfying the conditions.

Given $\delta_{1}, \delta_{2} \in \mathbb{R}_{+}^{X}\left(\mathbb{R}_{+}^{X}\right.$ stands for the set of all functions mapping $X$ into $\mathbb{R}_{+}$), we write

$$
\delta_{1} \leq_{\mathcal{I}} \delta_{2}
$$

provided $\delta_{1}(x) \leq \delta_{2}(x) \mathcal{I}$-(a.e.). Next, the symbol $\delta_{n} \rightarrow_{\mathcal{I}} \delta$ for $\delta_{n}, \delta \in \mathbb{R}_{+}^{X}$, $n \in \mathbb{N}_{0}$, means that $\lim _{n \rightarrow \infty} \delta_{n}(x)=\delta(x) \mathcal{I}$-(a.e.). It is easily seen that $\leq_{\mathcal{I}}$ is a transitive relation. From now on, $\mathbf{0}$ stands for the zero function in $\mathbb{R}_{+}^{X}$, i.e., $\mathbf{0}(x)=0$ for $x \in X$.

We need the following three hypotheses.

(H1) $\mathcal{I}$ is a $\sigma$-ideal in $X$ and $(Y, d)$ is a complete metric space.

(H2) Given $\Lambda: \mathbb{R}_{+}^{X} \rightarrow \mathbb{R}_{+}^{X}, \mathcal{T}: Y^{X} \rightarrow Y^{X}$ is $\Lambda$-contractive $\mathcal{I}$-(a.e.); i.e.,

$$
d(\mathcal{T} u, \mathcal{T} v) \leq_{\mathcal{I}} \Lambda \delta
$$

for every $u, v \in Y^{X}, \delta \in \mathbb{R}_{+}^{X}$ with $d(u, v) \leq_{\mathcal{I}} \delta$, where $d(u, v) \in \mathbb{R}_{+}^{X}$ is given by

$$
d(u, v)(x):=d(u(x), v(x))
$$

for $x \in X, u, v \in Y^{X}$.

(H3) If $\delta_{n} \in \mathbb{R}_{+}^{X}$ for $n \in \mathbb{N}$ and $\delta_{n} \rightarrow_{\mathcal{I}} \mathbf{0}$, then $\Lambda \delta_{n} \rightarrow_{\mathcal{I}} \mathbf{0 .}$

We define the notion $\rightarrow_{\mathcal{I}}$ in $Y^{X}$ analogously as in $\mathbb{R}_{+}^{X}$; that is, given $u, u_{n} \in Y^{X}$ for $n \in \mathbb{N}_{0}$ we write

$$
u_{n} \rightarrow_{\mathcal{I}} u
$$

provided $d\left(u_{n}, u\right) \rightarrow_{\mathcal{I}} \mathbf{0}$. Next, if $v_{1}, v_{2}$ map $X$ into a nonempty set $Z$, then $v_{1}=\mathcal{I} v_{2}$ means that $v_{1}(x)=v_{2}(x) \mathcal{I}$-(a.e.).

\section{A fixed point result}

Now, we present a fixed point theorem, which generalizes [10, Theorem 2.2] (see also $[5,6])$.

Theorem 2.1. Assume that hypotheses $(\mathrm{H} 1)-(\mathrm{H} 3)$ are valid. Suppose that there are functions $\varepsilon, \varepsilon^{*} \in \mathbb{R}_{+}^{X}$ and $f \in Y^{X}$ such that

$$
d(\mathcal{T} f, f) \leq_{\mathcal{I}} \varepsilon
$$

and

$$
\varepsilon^{*}={ }_{\mathcal{I}} \sum_{n=0}^{\infty} \Lambda^{n} \varepsilon .
$$

Then there exists a function $g: X \rightarrow Y$ satisfying the conditions

$$
\begin{gathered}
\mathcal{T}^{n} f \rightarrow_{\mathcal{I}} g, \\
\mathcal{T} g{ }_{\mathcal{I}} g, \\
d(f, g) \leq_{\mathcal{I}} \varepsilon^{*} .
\end{gathered}
$$


Moreover, if $\Lambda^{n} \varepsilon^{*} \rightarrow_{\mathcal{I}} \mathbf{0}$, then $g$ is an $\mathcal{I}$-unique function satisfying (2.4) and (2.5).

First, we prove an auxiliary lemma.

Lemma 2.2. Under the assumptions of Theorem 2.1, there exists a function $g: X \rightarrow Y$ such that (2.3) holds,

$$
d\left(\mathcal{T}^{m} f, g\right) \leq \sum_{k=m}^{\infty} \Lambda^{k} \varepsilon \quad \text { for } m \in \mathbb{N}_{0}
$$

and (2.4) is valid. Moreover, $g$ is an $\mathcal{I}$-unique function satisfying (2.6).

Proof. It is easy to show by induction that, for every $n \in \mathbb{N}_{0}$ there is $A_{n} \in \mathcal{I}$ such that

$$
d\left(\mathcal{T}^{n+1} f, \mathcal{T}^{n} f\right)(x) \leq\left(\Lambda^{n} \varepsilon\right)(x) \text { for } x \in X \backslash A_{n}
$$

(the case $n=0$ is trivial and the inductive step is a routine). Using the triangle inequality and (2.7), for every $n, m \in \mathbb{N}_{0}, n>m$, we have

$$
d\left(\mathcal{T}^{n} f, \mathcal{T}^{m} f\right)(x) \leq \sum_{k=m}^{n-1}\left(\Lambda^{k} \varepsilon\right)(x) \text { for } x \in X \backslash\left(\bigcup_{k=m}^{n-1} A_{k}\right) .
$$

Since (2.2) holds for $x \in X \backslash C$ with some $C \in \mathcal{I}$, it follows from the above estimate that $\left(\left(\mathcal{T}^{n} f\right)(x)\right)_{n \in \mathbb{N}}$ is a Cauchy sequence for every $x \in X \backslash D$, where

$$
D:=C \cup \bigcup_{k=0}^{\infty} A_{k} \in \mathcal{I} \text {. }
$$

As $(Y, d)$ is complete, the limit

$$
g(x):=\lim _{n \rightarrow \infty}\left(\mathcal{T}^{n} f\right)(x)
$$

exists for all $x \in X \backslash D$ and, letting $n \rightarrow \infty$ in (2.8), we obtain that (2.6) holds.

Let $g(x)=0$ for $x \in D$. We prove (2.4). Using (H3) with

$$
\delta_{n}(x):= \begin{cases}d\left(\mathcal{T}^{n} f, g\right)(x) & \text { for } x \in X \backslash D ; \\ 0 & \text { for } x \in D\end{cases}
$$

we have $\lim _{n \rightarrow \infty} \Lambda \delta_{n}(x)=0$ for $x \in X \backslash E$ with some $E \in \mathcal{I}$. By (H2), for every $n \in \mathbb{N}$ there exists $E_{n} \in \mathcal{I}$ such that

$$
d\left(\mathcal{T}^{n+1} f, \mathcal{T} g\right)(x) \leq\left(\Lambda \delta_{n}\right)(x) \text { for } x \in X \backslash E_{n} .
$$

It follows that

$$
\lim _{n \rightarrow \infty} d\left(\mathcal{T}^{n+1} f, \mathcal{T} g\right)(x)=0 \quad \text { for } x \in X_{0}:=X \backslash\left(\bigcup_{n \in \mathbb{N}} E_{n} \cup E\right) .
$$

Hence, in view of the definition of $g$,

$$
(\mathcal{T} g)(x)=g(x) \text { for } x \in X_{0} \backslash D .
$$


Suppose that $g_{1} \in Y^{X}$ also satisfies (2.6); i.e.,

$$
d\left(\mathcal{T}^{m} f, g_{1}\right) \leq_{\mathcal{I}} \sum_{k=m}^{\infty} \Lambda^{k} \varepsilon \quad \text { for } m \in \mathbb{N}_{0} .
$$

Clearly, there exists $D_{1} \in \mathcal{I}$ such that

$$
d\left(\mathcal{T}^{m} f, g_{1}\right)(x) \leq \sum_{k=m}^{\infty}\left(\Lambda^{k} \varepsilon\right)(x) \quad \text { for } x \in X \backslash D_{1}, m \in \mathbb{N}_{0} .
$$

Since $g$ satisfies (2.6), by the triangle inequality,

$$
d\left(g, g_{1}\right)(x) \leq 2 \sum_{k=m}^{\infty}\left(\Lambda^{k} \varepsilon\right)(x) \quad \text { for } x \in X_{1}:=X \backslash\left(D_{1} \cup D_{2}\right), m \in \mathbb{N}_{0},
$$

with some $D_{2} \in \mathcal{I}$. So, letting $m \rightarrow \infty$ and using (2.2) we obtain

$$
d\left(g_{1}, g\right)(x)=0 \quad \text { for } x \in X_{1} \backslash C .
$$

Thus $g=_{\mathcal{I}} g_{1}$, which completes the proof of the lemma.

Now we prove Theorem 2.1.

Proof of Theorem 2.1. In view of Lemma 2.2, there exists a function $g: X \rightarrow Y$ such that conditions (2.3) and (2.4) hold. Clearly, (2.5) is just (2.6) with $m=0$. It remains to show the statement on the $\mathcal{I}$-uniqueness.

To this end, assume that there is $A \in I$ with

$$
\lim _{n \rightarrow \infty} \Lambda^{n} \varepsilon^{*}(x)=0 \quad \text { for } x \in X \backslash A .
$$

Let $g_{1}: X \rightarrow Y$ be such that $\mathcal{T} g_{1}=\mathcal{I} g_{1}$ and $d\left(f, g_{1}\right) \leq_{\mathcal{I}} \varepsilon^{*}$. Since $\mathcal{T}$ is $\Lambda$-contractive, there exists $B \in \mathcal{I}$ such that

$$
d\left(\mathcal{T}^{n} f, \mathcal{T}^{n} g_{1}\right)(x) \leq\left(\Lambda^{n} \varepsilon^{*}\right)(x) \text { for } x \in X \backslash B, n \in \mathbb{N} .
$$

But there is $C \in \mathcal{I}$ such that $\mathcal{T}^{n} g_{1}(x)=g_{1}(x)$ for $x \in X \backslash C$ and $n \in \mathbb{N}$. So,

$$
d\left(\mathcal{T}^{n} f, g_{1}\right)(x) \leq\left(\Lambda^{n} \varepsilon^{*}\right)(x) \text { for } x \in X \backslash(B \cup C),
$$

whence letting $n \rightarrow \infty$, in view of $(2.3)$, we get $g=_{\mathcal{I}} g_{1}$, which ends the proof of Theorem 2.1.

Remark 2.3. Note that, if $\varepsilon, \varepsilon^{*} \in \mathbb{R}_{+}^{X}, \Lambda: \mathbb{R}_{+}^{X} \rightarrow \mathbb{R}_{+}^{X}$ is $\mathcal{I}$-additive (i.e., $\Lambda(u+v)=_{\mathcal{I}} \Lambda u+\Lambda v$ for $\left.u, v \in \mathbb{R}_{+}^{X}\right)$ and (H3) and (2.2) hold, then

$$
\Lambda^{n} \varepsilon^{*} \rightarrow_{\mathcal{I}} \mathbf{0} .
$$

In fact, in view of the additivity of $\Lambda$ and (H3), for every $k \in \mathbb{N}$ and a sequence $\left(\delta_{n}\right)_{n \in \mathbb{N}}$ of elements of $\mathbb{R}_{+}^{X}$ with $\delta_{n} \rightarrow_{\mathcal{I}} \delta$, we have

$$
\Lambda^{k}\left(\delta_{n}-\delta\right) \rightarrow_{\mathcal{I}} \mathbf{0}
$$

and consequently $\Lambda^{k} \delta_{n} \rightarrow_{\mathcal{I}} \Lambda^{k} \delta$. Therefore, for each $k \in \mathbb{N}$,

$$
\Lambda^{k} \varepsilon^{*}(x)=\sum_{i=0}^{\infty} \Lambda^{k+i} \varepsilon(x)=\sum_{i=k}^{\infty} \Lambda^{i} \varepsilon(x) \quad \mathcal{I} \text {-(a.e.). }
$$


Thus, by (2.2),

$$
\lim _{n \rightarrow \infty} \Lambda^{n} \varepsilon^{*}(x)=0 \quad \mathcal{I} \text {-(a.e.). }
$$

From Theorem 2.1 we can obtain the following generalization of $[6$, Theorem 1] (cf. also [9, Theorem 1]). (We say that $\Lambda: \mathbb{R}^{X} \rightarrow \mathbb{R}^{X}$ is $\mathcal{I}$ nondecreasing if $\Lambda \delta_{1} \leq_{\mathcal{I}} \Lambda \delta_{2}$ for every $\delta_{1}, \delta_{2} \in \mathbb{R}^{X}$ such that $\delta_{1} \leq_{\mathcal{I}} \delta_{2}$.)

Corollary 2.4. Let hypotheses (H1) and (H3) be valid and let $\Lambda$ be $\mathcal{I}$-nondecreasing. Assume that $\mathcal{T}: Y^{X} \rightarrow Y^{X}$ is an operator fulfilling the inequality

$$
d(\mathcal{T} u, \mathcal{T} v) \leq_{\mathcal{I}} \Lambda d(u, v) \quad \text { for } u, v \in Y^{X},
$$

and functions $\varepsilon^{*}, \varepsilon \in \mathbb{R}_{+}^{X}$ and $f \in Y^{X}$ are such that conditions (2.1) and (2.2) hold. Then there exists a function $g: X \rightarrow Y$ satisfying conditions (2.3), (2.4) and (2.5). If additionally $\Lambda^{n} \varepsilon^{*} \rightarrow_{\mathcal{I}} \mathbf{0}$, then $g$ is an $\mathcal{I}$-unique function satisfying (2.4) and (2.5).

Proof. Take $u, v \in Y^{X}$ and $\delta \in \mathbb{R}_{+}^{X}$ satisfying the condition

$$
d(u, v) \leq_{\mathcal{I}} \delta
$$

Since $\Lambda$ is $\mathcal{I}$-nondecreasing, in view of (2.9) we have

$$
d(\mathcal{T} u, \mathcal{T} v) \leq_{\mathcal{I}} \Lambda d(u, v) \leq_{\mathcal{I}} \Lambda \delta .
$$

Thus we have proved that (H2) holds. Consequently, Theorem 2.1 completes the proof.

Theorem 2.1 also implies a generalization of [5, Theorem 1]. To present it we need the following hypotheses.

(H4) $L_{1}, \ldots, L_{k} \in \mathbb{R}_{+}^{X}, h_{1}, \ldots, h_{k} \in X^{X}$ are given maps such that

$$
h_{i}^{-1}(I) \in \mathcal{I} \quad \text { for } I \in \mathcal{I}, i \in\{1, \ldots, k\} .
$$

(H5) $\mathcal{T}: Y^{X} \rightarrow Y^{X}$ is an operator such that, for any $u, v \in Y^{X}$,

$$
d((\mathcal{T} u)(x),(\mathcal{T} v)(x)) \leq \sum_{i=1}^{k} L_{i}(x) d\left(u\left(h_{i}(x)\right), v\left(h_{i}(x)\right)\right) \quad \mathcal{I} \text {-(a.e.). }
$$

(H6) $\Lambda: \mathbb{R}_{+}^{X} \rightarrow \mathbb{R}_{+}^{X}$ is defined by

$$
(\Lambda \delta)(x):=\sum_{i=1}^{k} L_{i}(x) \delta\left(h_{i}(x)\right) \quad \text { for } \delta \in \mathbb{R}_{+}^{X}, x \in X .
$$

Corollary 2.5. Assume that hypotheses (H1) and (H4)-(H6) are satisfied. Suppose that there are functions $\varepsilon^{*}, \varepsilon \in \mathbb{R}_{+}^{X}$ and $f \in Y^{X}$ such that conditions (2.1) and (2.2) hold. Then there exists an $\mathcal{I}$-unique function $g: X \rightarrow Y$ satisfying (2.4) and (2.5). Moreover, (2.3) holds.

Proof. Clearly, $\Lambda$ is $\mathcal{I}$-nondecreasing, because each $L_{i}$ is nonnegative. Take any sequence $\left(\delta_{n}\right)_{n \in \mathbb{N}}$ in $\mathbb{R}_{+}^{X}$ such that there exists $A \in \mathcal{I}$ with

$$
\lim _{n \rightarrow \infty} \delta_{n}(x)=0 \quad \text { for } x \in X \backslash A \text {. }
$$


It is easily seen that

$$
\lim _{n \rightarrow \infty} \delta_{n}\left(h_{i}(x)\right)=0 \quad \text { for } x \in X \backslash h_{i}^{-1}(A), i \in\{1, \ldots, k\},
$$

and consequently,

$$
\lim _{n \rightarrow \infty}\left(\Lambda \delta_{n}\right)(x)=0 \quad \text { for } x \in X \backslash \bigcup_{i=1}^{k} h_{i}^{-1}(A) .
$$

Since, according to $(\mathrm{H} 4), h_{i}^{-1}(A) \in \mathcal{I}$ for $i \in\{1, \ldots, k\}$, this means that we have proved (H3).

Next,

$$
\sum_{i=1}^{k} L_{i}(x) d\left(u\left(h_{i}(x)\right), v\left(h_{i}(x)\right)\right)=\Lambda d(u, v)(x) \quad \text { for } u, v \in Y^{X}, x \in X,
$$

whence (2.9) follows from (H5). Note yet that, by Remark 2.3, $\Lambda^{n} \varepsilon^{*} \rightarrow_{\mathcal{I}} \mathbf{0}$. So, the proof can be completed by applying Corollary 2.4 .

If we take $\mathcal{I}=\{\emptyset\}$, then Corollary 2.5 becomes [5, Theorem 1$]$.

\section{Applications in Ulam's type stability}

Given $L_{1}, \ldots, L_{k} \in R_{+}^{X}$ and $h_{1}, \ldots, h_{k} \in X^{X}$, we introduce yet the following hypothesis.

(H7) $\Phi: X \times Y^{k} \rightarrow Y$ is such that, for every $\left(y_{1}, \ldots, y_{k}\right),\left(z_{1}, \ldots, z_{k}\right) \in Y^{k}$,

$$
d\left(\Phi\left(x, y_{1}, \ldots, y_{k}\right), \Phi\left(x, z_{1}, \ldots, z_{k}\right)\right) \leq \sum_{i=1}^{k} L_{i}(x) d\left(y_{i}, z_{i}\right) \quad \mathcal{I} \text {-(a.e.). }
$$

Corollary 2.5 yields at once the following two outcomes that generalize (at least to some extent) several results in $[1,2,5,7,8,13,18,19]$.

Corollary 3.1. Assume that hypotheses (H1), (H4), (H6) and (H7) hold. Let $\varepsilon^{*}, \varepsilon: X \rightarrow \mathbb{R}_{+}$satisfy $(2.2)$ and let $f \in Y^{X}$ be such that

$$
d\left(f(x), \Phi\left(x, f\left(h_{1}(x)\right), \ldots, f\left(h_{k}(x)\right)\right)\right) \leq \varepsilon(x) \quad \mathcal{I} \text {-(a.e.). }
$$

Then there exists an $\mathcal{I}$-unique function $g: X \rightarrow Y$ such that (2.5) is valid and

$$
\Phi\left(x, g\left(h_{1}(x)\right), \ldots, g\left(h_{k}(x)\right)\right)=g(x) \quad \mathcal{I} \text {-(a.e.). }
$$

Moreover, (2.3) holds, with $\mathcal{T}: Y^{X} \rightarrow Y^{X}$ defined by

$$
\mathcal{T} u(x):=\Phi\left(x, u\left(h_{1}(x)\right), \ldots, u\left(h_{k}(x)\right)\right) \quad \text { for } u \in Y^{X}, x \in X .
$$

Proof. By hypothesis (H7), the operator $\mathcal{T}: Y^{X} \rightarrow Y^{X}$ defined by (3.3) satisfies (H5). Inequality (3.1) immediately yields (2.1). To end the proof we only need to apply Corollary 2.5.

Remark 3.2. Observe that if we assume in Corollary 3.1 that, additionally, $(X, \mathcal{M})$ and $(Y, \mathcal{N})$ are measurable spaces, $\mathcal{I} \subset \mathcal{M}$ and $\mathcal{T}^{n} f$ is $(\mathcal{M}, \mathcal{N})$ measurable for each $n \in \mathbb{N}$, then $g$ is $(\mathcal{M}, \mathcal{N})$-measurable, because it is a pointwise limit of a sequence of $(\mathcal{M}, \mathcal{N})$-measurable functions. 
Corollary 3.3. Assume that hypotheses $(\mathrm{H} 1),(\mathrm{H} 4)$ and $(\mathrm{H} 7)$ are valid. Let

$$
q:=\sum_{i=1}^{k} L_{i}, \quad q(x)<1, \quad \mathcal{I} \text {-(a.e.) }
$$

and let $\varepsilon: X \rightarrow \mathbb{R}_{+}$be such that

$$
\varepsilon \circ h_{i} \leq_{\mathcal{I}} \varepsilon, \quad q \circ h_{i} \leq_{\mathcal{I}} q \quad \text { for } i \in\{1, \ldots, k\} .
$$

If $f \in Y^{X}$ satisfies (3.1), then there exists an $\mathcal{I}$-unique function $g: X \rightarrow Y$ satisfying (3.2) and

$$
d(f, g)(x) \leq \frac{\varepsilon(x)}{1-q(x)} \quad \mathcal{I} \text {-(a.e.). }
$$

Moreover, (2.3) holds, with $\mathcal{T}$ defined by (3.3).

Proof. Assume that $A_{1}, \ldots, A_{k}, B_{1}, \ldots, B_{k}, D \in \mathcal{I}$ are such that $q(x)<1$ for $x \in X \backslash D$,

$$
\begin{array}{ll}
\varepsilon\left(h_{i}(x)\right) \leq \varepsilon(x) & \text { for } x \in X \backslash A_{i}, i \in\{1, \ldots, k\}, \\
q\left(h_{i}(x)\right) \leq q(x) & \text { for } x \in X \backslash B_{i}, i \in\{1, \ldots, k\} .
\end{array}
$$

Put

$$
C=D \cup \bigcup_{i=1}^{k}\left(A_{i} \cup B_{i}\right)
$$

and define $\Lambda: \mathbb{R}_{+}^{X} \rightarrow \mathbb{R}_{+}^{X}$ as in (H6). We prove by induction that

$$
\Lambda^{n} \varepsilon(x) \leq q(x)^{n} \varepsilon(x) \text { for } x \in X \backslash C, n \in \mathbb{N}_{0} .
$$

This statement is trivial for $n=0$. Assume (3.6) for a fixed $n \geq 0$ and take $x \in X \backslash C$. The definition of $\Lambda$ yields

$$
\Lambda^{n+1} \varepsilon(x)=\sum_{i=1}^{k} L_{i}(x)\left(\Lambda^{n} \varepsilon\right)\left(h_{i}(x)\right) \leq \sum_{i=1}^{k} L_{i}(x)\left(q\left(h_{i}(x)\right)\right)^{n} \varepsilon\left(h_{i}(x)\right)
$$

and, by (3.4) and (3.5), we get

$$
\left(\Lambda^{n+1} \varepsilon\right)(x) \leq \sum_{i=1}^{k} L_{i}(x)(q(x))^{n} \varepsilon(x)=q(x)^{n+1} \varepsilon(x) .
$$

Thus we have proved (3.6). Consequently,

$$
\sum_{n=0}^{\infty} \Lambda^{n} \varepsilon(x) \leq \sum_{n=0}^{\infty} q(x)^{n} \varepsilon(x)=\frac{\varepsilon(x)}{1-q(x)}, \quad x \in X \backslash C .
$$

Hence condition (2.2) holds with some $\varepsilon^{*} \in \mathbb{R}_{+}^{X}$ such that $\varepsilon^{*}={ }_{\mathcal{I}} \sum_{n=0}^{\infty} \Lambda^{n} \varepsilon$ and

$$
\varepsilon^{*}(x) \leq \frac{\varepsilon(x)}{1-q(x)}, \quad x \in X .
$$

So, the statement follows from Corollary 3.1. Moreover, we can deduce the uniqueness from (3.6) (putting $\varepsilon(x) /(1-q(x))$ in place of $\varepsilon(x)$ ), analogously as in the proof of Theorem 2.1 (putting $\varepsilon(x) /(1-q(x))$ in place of $\varepsilon^{*}(x)$ ). 
Finally, we present an application of Theorem 2.1 in the investigation of stability of a particular functional equation, namely the polynomial functional equation. We generalize [9, Theorem 2] in this way.

Theorem 3.4. Let $B$ be a Banach commutative algebra with the unit element denoted by $e, m \in \mathbb{N} \backslash\{1\}, \xi_{1}, \ldots, \xi_{m} \in X^{X}$, and let $\mu \in X^{X}$ be a bijection such that $\mu(I), \xi^{-1}(I) \in \mathcal{I}$ for each $I \in \mathcal{I}, i \in\{1, \ldots, m\}, r>0$, $p:\{1, \ldots, m\} \rightarrow \mathbb{N}, G, a_{1}, \ldots, a_{m} \in B^{X}$,

$$
\begin{gathered}
\left.\sum_{i=1}^{m}\left\|a_{i}\left(\mu^{-1}(x)\right)\right\| r^{p(i)}+\left\|G\left(\mu^{-1}(x)\right)\right\| \leq r \quad \mathcal{I} \text {-(a.e. }\right), \\
\Lambda \eta(x):=\sum_{i=1}^{m} p(i) r^{p(i)-1}\left\|a_{i}\left(\mu^{-1}(x)\right)\right\| \eta\left(\xi_{i}\left(\mu^{-1}(x)\right)\right), \quad \eta \in \mathbb{R}_{+}^{X}, x \in X .
\end{gathered}
$$

If $\delta, \chi \in \mathbb{R}_{+}^{X}$ and $f \in \mathcal{B}_{r}:=\left\{h \in B^{X}:\|h(x)\| \leq r\right\}$ satisfy

$$
\begin{gathered}
\left\|f(\mu(x))+\sum_{i=1}^{m} a_{i}(x) f\left(\xi_{i}(x)\right)^{p(i)}-G(x)\right\| \leq \delta(x) \quad \mathcal{I} \text {-(a.e.) }, \\
\chi(x):=\sum_{n=0}^{\infty} \Lambda^{n} \delta\left(\mu^{-1}(x)\right) \quad \mathcal{I} \text {-(a.e.), }
\end{gathered}
$$

then there exists an $\mathcal{I}$-unique function $g \in \mathcal{B}_{r}$ such that

$$
g(\mu(x))+\sum_{j=1}^{m} a_{j}(x) g\left(\xi_{j}(x)\right)^{p(j)}=G(x) \quad \mathcal{I} \text {-(a.e.) }
$$

and

$$
\|g(x)-f(x)\| \leq \chi(x) \quad \mathcal{I} \text {-(a.e.). }
$$

Proof. Set $Y:=\{b \in B:\|b\| \leq r\}$. Clearly $Y$ is a complete metric space with the metric $d$ given by

$$
d\left(y_{1}, y_{2}\right)=\left\|y_{1}-y_{2}\right\|, \quad y_{1}, y_{2} \in Y .
$$

Define $\mathcal{T}: Y^{X} \rightarrow Y^{X}$ by (3.3) with $h_{i}:=\xi_{i} \circ \mu^{-1}$ for $i \in\{1, \ldots, m\}$ and $\Phi: X \times Y^{m} \rightarrow Y$ given by

$$
\Phi\left(x, y_{1}, \ldots, y_{m}\right):=G\left(\mu^{-1}(x)\right)-\sum_{i=1}^{m} a_{i}\left(\mu^{-1}(x)\right) y_{i}^{p(i)}
$$

for $x \in X,\left(y_{1}, \ldots, y_{m}\right) \in Y^{m}$. Let $\Lambda$ be defined as in (H6) with

$$
L_{i}(x):=p(i) r^{p(i)-1}\left\|a_{i}\left(\mu^{-1}(x)\right)\right\| \text { for } i \in\{1, \ldots, m\} .
$$

It is easily seen that, by our assumptions, (H4) is valid and, in view of (3.7) and (3.8), conditions (2.2) and (3.1) hold with $\varepsilon:=\delta \circ \mu^{-1}$ and $\varepsilon^{*}:=\chi$.

Now, we prove that (H7) is fulfilled. Clearly, for every $y, z \in Y$ and $k \in\{1, \ldots, m\}$ we have

$$
\left\|y^{k}-z^{k}\right\| \leq\|y-z\| \sum_{j=0}^{k-1}\|y\|^{j}\|z\|^{k-j-1} \leq\|y-z\| k r^{k-1} .
$$


Consequently, for $x \in X$ and $y_{1}, \ldots, y_{m}, z_{1}, \ldots, z_{m} \in Y$,

$$
\begin{aligned}
d\left(\Phi\left(x, y_{1}, \ldots, y_{m}\right), \Phi\left(x, z_{1}, \ldots, z_{m}\right)\right) & \\
& \leq \sum_{i=1}^{m}\left\|a_{i}\left(\mu^{-1}(x)\right)\right\|\left\|y_{i}^{p(i)}-z_{i}^{p(i)}\right\| \\
& \leq \sum_{i=1}^{m}\left\|a_{i}\left(\mu^{-1}(x)\right)\right\|\left\|y_{i}-z_{i}\right\| p(i) r^{p(i)-1} \\
& =\sum_{i=1}^{m} L_{i}(x) d\left(y_{i}, z_{i}\right)
\end{aligned}
$$

whence (H7) is valid. Now, applying Corollary 3.1, we obtain our assertion.

\section{References}

[1] R. P. Agarwal, B. Xu and W. Zhang, Stability of functional equations in single variable. J. Math. Anal. Appl. 288 (2003), 852-869.

[2] J. A. Baker, The stability of certain functional equations. Proc. Amer. Math. Soc. 112 (1991), 729-732.

[3] N. Brillouët-Belluot, J. Brzdęk and K. Ciepliński, On some recent developments in Ulam's type stability. Abstr. Appl. Anal. 2012 (2012), Art. ID 716936, 41 pages.

[4] J. Brzdęk, L. Cădariu and K. Ciepliński, Fixed point theory and the Ulam stability. J. Funct. Spaces 2014 (2014), Article ID 829419, 16 pages.

[5] J. Brzdęk, J. Chudziak and Z. Páles, A fixed point approach to stability of functional equations. Nonlinear Anal. 74 (2011), 6728-6732.

[6] J. Brzdęk and K. Ciepliński, A fixed point approach to the stability of functional equations in non-Aarchimedean metric spaces. Nonlinear Anal. 74 (2011), 68616867.

[7] J. Brzdęk, D. Popa and B. Xu, The Hyers-Ulam stability of linear equations of higher orders. Acta Math. Hungar. 120 (2008), 1-8.

[8] J. Brzdęk, D. Popa and B. Xu, Remarks on stability of the linear recurrence of higher order. Appl. Math. Lett. 23 (2010), 1459-1463.

[9] J. Brzdęk and S. Stević, A note on stability of polynomial equations. Aequationes Math. 85 (2013), 519-527.

[10] L. Cădariu, L. Găvruţa and P. Găvruţa, Fixed points and generalized HyersUlam stability. Abstr. Appl. Anal. 2012 (2012), Article ID 712743, 10 pages.

[11] S. Czerwik, Functional Equations and Inequalities in Several Variables. World Scientific, London, 2002.

[12] G. L. Forti, Hyers-Ulam stability of functional equations in several variables. Aequationes Math. 50 (1995), 143-190.

[13] G. L. Forti, Comments on the core of the direct method for proving Hyers-Ulam stability of functional equations. J. Math. Anal. Appl. 295 (2004), 127-133.

[14] D. H. Hyers, On the stability of the linear functional equation. Proc. Natl. Acad. Sci. USA 27 (1941), 222-224. 
[15] D. H. Hyers, G. Isac and Th. M. Rassias, Stability of Functional Equations in Several Variables. Birkhäuser Boston, Boston, MA, 1998.

[16] S.-M. Jung, Hyers-Ulam-Rassias Stability of Functional Equations in Mathematical Analysis. Hadronic Press, Palm Harbor, FL, 2001.

[17] Z. Moszner, On the stability of functional equations. Aequationes Math. 77 (2009), 33-88.

[18] T. Trif, On the stability of a general gamma-type functional equation. Publ. Math. Debrecen 60 (2002), 47-61.

[19] T. Trif, Hyers-Ulam-Rassias stability of a linear functional equation with constant coefficients. Nonlinear Funct. Anal. Appl. 5 (2006), 881-889.

[20] S. M. Ulam, A Collection of Mathematical Problems. Interscience Publishers, New York, 1960. Reprinted as: Problems in Modern Mathematics. John Wiley \& Sons, New York, 1964.

\author{
Anna Bahyrycz \\ Department of Mathematics \\ Pedagogical University \\ Podchorążych 2 \\ 30-084 Kraków \\ Poland \\ e-mail: bah@up.krakow.pl \\ Janusz Brzdęk \\ Department of Mathematics \\ Pedagogical University \\ Podchorążych 2 \\ 30-084 Kraków \\ Poland \\ e-mail: jbrzdek@up.krakow.pl \\ Eliza Jabłońska \\ Department of Mathematics \\ Rzeszów University of Technology \\ Powstańców Warszawy 12 \\ 35-959 Rzeszów \\ Poland \\ e-mail: elizapie@prz.edu.pl \\ Jolanta Olko \\ Faculty of Applied Mathematics \\ AGH University of Science and Technology \\ Mickiewicza 30 \\ 30-059 Kraków \\ Poland
}

Open Access This article is distributed under the terms of the Creative Commons Attribution 4.0 International License (http://creativecommons.org/licenses/by/4.0/), which permits unrestricted use, distribution, and reproduction in any medium, provided you give appropriate credit to the original author(s) and the source, provide a link to the Creative Commons license, and indicate if changes were made. 